\title{
Frequency of Screening and SBT Technique Trial - North American Weaning Collaboration (FAST-NAWC): a protocol for a multicenter, factorial randomized trial
}

K. E. A. Burns ${ }^{1,2,3^{*}}$ (D), Leena Rizvi ${ }^{2}$, Deborah J. Cook ${ }^{4}$, Andrew J. E. Seely ${ }^{5}$, Bram Rochwerg ${ }^{4,6}$, Francois Lamontagne ${ }^{7}$, John W. Devlin ${ }^{8,9}$, Peter Dodek ${ }^{10,11}$, Michael Mayette ${ }^{7}$, Maged Tanios ${ }^{12}$, Audrey Gouskos ${ }^{13}$, Phyllis Kay ${ }^{13}$, Susan Mitchell ${ }^{13}$, Kenneth C. Kiedrowski ${ }^{13}$, Nicholas S. Hill ${ }^{9}$ and For the Canadian Critical Care Trials Group

\begin{abstract}
Rationale: In critically ill patients receiving invasive mechanical ventilation (MV), research supports the use of daily screening to identify patients who are ready to undergo a spontaneous breathing trial (SBT) followed by conduct of an SBT. However, once daily (OD) screening is poorly aligned with the continuous care provided in most intensive care units (ICUs) and the best SBT technique for clinicians to use remains controversial.

Objectives: To identify the optimal screening frequency and SBT technique to wean critically ill adults in the ICU.

Methods: We aim to conduct a multicenter, factorial design randomized controlled trial with concealed allocation, comparing the effect of both screening frequency (once versus at least twice daily [ALTD]) and SBT technique (Pressure Support [PS] + Positive End-Expiratory Pressure [PEEP] vs T-piece) on the time to successful extubation (primary outcome) in 760 critically ill adults who are invasively ventilated for at least $24 \mathrm{~h}$ in 20 North American ICUs. In the OD arm, respiratory therapists (RTs) will screen study patients between 06:00 and 08:00 $\mathrm{h}$. In the ALTD arm, patients will be screened at least twice daily between 06:00 and 08:00 $\mathrm{h}$ and between 13:00 and 15:00 $\mathrm{h}$ with additional screens permitted at the clinician's discretion. When the SBT screen is passed, an SBT will be conducted using the assigned technique (PS + PEEP or T-piece). We will follow patients until successful extubation, death, ICU discharge, or until day 60 after randomization. We will contact patients or their surrogates six months after randomization to assess health-related quality of life and functional status.
\end{abstract}

Relevance: The around-the-clock availability of RTs in North American ICUs presents an important opportunity to identify the optimal SBT screening frequency and SBT technique to minimize patients' exposure to invasive ventilation and ventilator-related complications.

Trial registration: Clinical Trials.gov, NCT02399267. Registered on Nov 21, 2016 first registered.

Keywords: Weaning, Spontaneous breathing trial, Screening, Randomized controlled trial, Successful extubation

\footnotetext{
* Correspondence: burnsk@smh.ca

${ }^{1}$ Interdepartmental Division of Critical Care, University of Toronto, Toronto,

ON, Canada

${ }^{2}$ Department of Medicine, Division of Critical Care Medicine, St Michael's

Hospital, 30 Bond Street, Office 4-045 Donnelly Wing, Toronto, ON M5B 1W8,

Canada

Full list of author information is available at the end of the article
}

(c) The Author(s). 2019 Open Access This article is distributed under the terms of the Creative Commons Attribution 4.0 International License (http://creativecommons.org/licenses/by/4.0/), which permits unrestricted use, distribution, and reproduction in any medium, provided you give appropriate credit to the original author(s) and the source, provide a link to the Creative Commons license, and indicate if changes were made. The Creative Commons Public Domain Dedication waiver (http://creativecommons.org/publicdomain/zero/1.0/) applies to the data made available in this article, unless otherwise stated. 


\section{Introduction}

Weaning from invasive mechanical ventilation (MV) is the process during which the work of breathing is transferred from the ventilator back to the patient. Nearly $40 \%$ of the time spent on invasive MV is dedicated to weaning $[1,2]$. Although invasive MV is effective in managing respiratory failure, its use is associated with the development of numerous complications including ventilator-associated pneumonia (VAP) and respiratory muscle weakness [3]. The risk for VAP increases after the fifth day of invasive $\mathrm{MV}$, is associated with substantial morbidity, and may increase mortality [4]. Conversely, premature or failed attempts at extubation necessitating reintubation are also associated with greater risk of VAP [5], prolonged intensive care unit (ICU) stay, and increased mortality $[6,7]$. Consequently, in their efforts to minimize patient's exposure to invasive MV, clinicians are challenged by a "tradeoff" between the complications associated with protracted ventilation and the risks associated with failed attempts at extubation [8].

More than two decades of research support the use of specific strategies to limit invasive MV including: (1) the use of multidisciplinary screening protocols to identify appropriate candidates for a spontaneous breathing trial (SBT) $[9,10]$; (2) the conduct of SBTs $[6,7,11,12]$ in patients who pass screening criteria; and (3) the use of specific modes and techniques (reductions in Pressure Support [PS]) and once daily (OD) SBTs (PS \pm positive end expiratory pressure [PEEP] or T-piece) $[12-14]$ to discontinue ventilator support in patients who fail an initial SBT. In a 2014 Cochrane review of 17 RCTs $(n=$ 2434), use of an SBT screening protocol compared to usual care was associated with significant reductions in weaning time, duration of MV, and ICU stay [9]. However, the strength of the conclusions that could be made from this meta-analysis were limited by heterogeneous populations, individual study risk of bias, and comparison of OD screening (intervention arm) to usual care (control arm) in most included trials. Importantly, no trial in this review compared more frequent screening to daily screening. Only one trial $(n=385)$ compared twice daily screening to usual care and noted a significantly shorter duration of MV and a trend toward a lower VAP with twice daily screening [15]. In national and international weaning surveys, daily SBT screening is the current standard of care [16, 17]. Notwithstanding, daily screening may be poorly aligned with the continuous care provided in most ICUs because it is not patientcentered and disregards the impact that treatment interventions, initiated after morning patient care rounds (e.g. reducing sedation), may have on SBT screening efforts later in the day.

The preferred technique to conduct an SBT remains unclear. Although PS SBTs are more commonly used in
North America, significant inter-institutional variability exists in how SBTs are conducted [18]. Two metaanalyses of randomized controlled trials (RCTs) compared PS and T-piece weaning, including, but not limited to, the conduct of SBTs, found beneficial effects of PS weaning $[19,20]$. Similarly, a meta-analysis that directly compared alternative SBT techniques [11] and two guidance documents $[12,21]$ support use of PS SBTs. Conversely, a physiologic meta-analysis favored use of T-piece SBTs [22].

Although a large body of evidence regarding weaning and SBT conduct exists, it remains insufficient to guide care regarding how frequent SBT screening should occur and the SBT technique that should be used. The around-the-clock availability of respiratory therapists (RTs) in most North American ICUs presents a unique opportunity to identify the optimal SBT screening frequency and SBT technique. In the Frequency of Screening and SBT Technique - North American Weaning Collaborative (FAST-NAWC) Trial, we will compare the effect of different screening frequencies (OD vs at least twice daily [ALTD]) and SBT techniques (PS + PEEP vs T-piece) in critically ill adults on time to successful extubation [23].

\section{Objectives}

\section{Primary objectives}

The primary objectives of the FAST-NAWC trial are to demonstrate the effect of the alternative:

1. different screening frequencies (OD vs ALTD) on time to successful extubation;

2. different SBT techniques (PS + PEEP vs T-piece) on time to successful extubation.

\section{Secondary objectives}

We will obtain estimates of the impact of the alternative screening and SBT techniques on other clinically important outcomes (see "Secondary outcomes" below).

\section{Methods}

\section{Study population}

We will include 760 critically ill adults aged $\geq 18$ years (USA) or $\geq 16$ years (Canada) or admitted to an adult ICU in approximately 20 ICUs in North America.

\section{Eligibility}

We will include critically ill adults who: (1) have received invasive mechanical ventilation for $\geq 24 \mathrm{~h}$; (2) are capable of initiating spontaneous breaths or triggering the ventilator to give a breath on ventilator modes commonly used in the ICU; (3) require a fractional concentration of inspired oxygen $\left(\mathrm{FiO}_{2}\right) \leq 70 \%$; and (4) PEEP $\leq$ 
$12 \mathrm{~cm} \mathrm{H}_{2} \mathrm{O}$. We will exclude patients who meet one or more of the exclusion criteria listed in Table 1.

\section{Enrollment}

Research personnel (research coordinators and/or RTs) will identify, consent, and enroll eligible patients from Monday to Friday during regular hours using a central randomization system, stratified by ICU with variable undisclosed block sizes. With the factorial design, patients will be randomized to both a screening frequency (OD vs ALTD) and an SBT technique (PS + PEEP vs Tpiece) (Fig. 1).

\section{Informed consent}

This protocol was approved by the research ethics board of St. Michael's Hospital (Toronto) and of participating ICUs. Given the minimal risk associated with the interventions being evaluated and the need to enroll patients

Table 1 Exclusion criteria

1. Brain death or expected brain death

2. Patients who have evidence of myocardial ischemia in the $24-h$ period before enrollment, except if current trend in troponin is downward AND it has been $\geq 24 \mathrm{~h}$ since last troponin peak or the patient has undergone a revascularization procedure and attending physician has no concerns regarding ongoing ischemia

3. Patients who have received continuous invasive mechanical ventilation for $\geq 2$ weeks

4. Patients who have a tracheostomy in situ at the time of screening

5. Patients who are receiving sedative infusions for seizures or alcohol withdrawal

6. Patients who require escalating doses of sedative agents

7. Patients who are receiving neuromuscular blockers or who have known quadriplegia, paraplegia, or four-limb weakness or paralysis preventing active mobilization (e.g. active range of motion, exercises in bed, sitting at edge of bed, transferring from bed to chair, standing, marching in place, ambulating)

8. Patients who are moribund (e.g. at imminent risk for death) or who have limitations of treatment (e.g. withdrawal of support, do not reintubate order, however, do not resuscitate orders will be permitted)

9. Patients who have profound neurologic deficits (e.g. after cardiac or respiratory arrest, large intracranial stroke or bleed) or GCS $\leq 6$

10. Patients who are using modes that automate SBT conduct

11. Patients who are current enrolled in a confounding study that includes a weaning protocol, or

12. Patients who were previously enrolled in this trial

13. Patients who have already undergone an SBT or are on T-piece, or CPAP alone (without PS), or PS $\leq 8 \mathrm{~cm} \mathrm{H} \mathrm{H}_{2} \mathrm{O}$ regardless of PEEP, or other "SBT equivalent" settings immediately before randomization

14. Patients who have already undergone extubation (planned, unplanned [e.g. self, accidental]) during the same ICU admission

PS Pressure Support, PAV Proportional Assist Ventilation, AC assist control, SIMV synchronized intermittent mandatory ventilation, $P R V C$ pressure regulated volume control, VS volume support, $A P R V$ airway pressure release ventilation, $\mathrm{FiO}_{2}$ inspired fractional concentration of oxygen, $P E E P$ positive endexpiratory pressure, GCS Glasgow Coma Scale, SBT spontaneous breathing trial, CPAP continuous positive airway pressure as soon as possible after they can either initiate spontaneous breaths or trigger breaths, we will request ethics approval to use a hybrid consent model that prioritizes obtaining consent from patients (with decision-making capacity) or SDMs (when available) and permits deferred consent in their absence. For patients who are enrolled under deferred consent [24], research personnel will obtain consent as soon as possible after randomization. We have used this hybrid consent model in two multicenter, pilot, screening frequency trials comparing OD and ALTD screening [25].

\section{Study interventions \\ Screening for readiness to undergo a spontaneous breathing trial}

In the OD arm, RTs will screen study patients daily between 06:00 and 08:00 h. In the ALTD arm, patients will be screened at least twice daily between 06:00 and 08:00 h and between 13:00 and 15:00 h; additional screening will be permitted at the discretion of the ICU team. If a screening period is missed (inadvertently or due to an operation/procedure necessitating absence from the ICU), it may be conducted later on the same day and ideally within $6 \mathrm{~h}$ of the scheduled screening period. For patients randomized after 10:00 h, only one screening assessment will be required on the first study day regardless of study arm.

To pass a screening assessment and undergo an SBT, all of the following criteria must be met:

1. The patient must be capable of initiating spontaneous breaths on PS or Proportional Assist Ventilation (PAV) or triggering breaths on volume or pressure Assist Control (AC), volume or pressure synchronized intermittent mandatory ventilation $(\mathrm{SIMV}) \pm \mathrm{PS}$, pressure regulated volume control (PRVC), volume support (VS), or Airway pressure regulated volume (APRV);

2. The ratio of partial pressure of oxygen to $\mathrm{FiO}_{2}$ $\left(\mathrm{PaO}_{2} / \mathrm{FiO}_{2}\right) \geq 200 \mathrm{mmHg}$;

3. Respiratory rate $\leq 35$ breaths $/ \mathrm{min}$;

4. $\mathrm{PEEP} \leq 10 \mathrm{~cm} \mathrm{H}_{2} \mathrm{O}$;

5. Heart rate $\leq 140$ beats $/ \mathrm{min}$;

6. The ratio of respiratory frequency to tidal volume $(\mathrm{f} / \mathrm{VT})<105$ breaths/min/L [26] during a $2 \mathrm{~min}$ assessment on Continuous positive airway pressure (CPAP) of $0 \mathrm{~cm} \mathrm{H}_{2} \mathrm{O}$ (alternatively PS $=0 \mathrm{~cm} \mathrm{H}_{2} \mathrm{O}$ /PEEP $=0 \mathrm{~cm} \mathrm{H}_{2} \mathrm{O}$ ).

\section{Conduct of spontaneous breathing trials}

After passing a screening assessment, patients will undergo an initial SBT according to treatment assignment (PS + PEEP vs T-piece). All SBTs will be $30-120$ min in duration with the actual duration selected by clinicians $[7,21]$. SBTs will be conducted on T-piece (off ventilator with no CPAP/ 


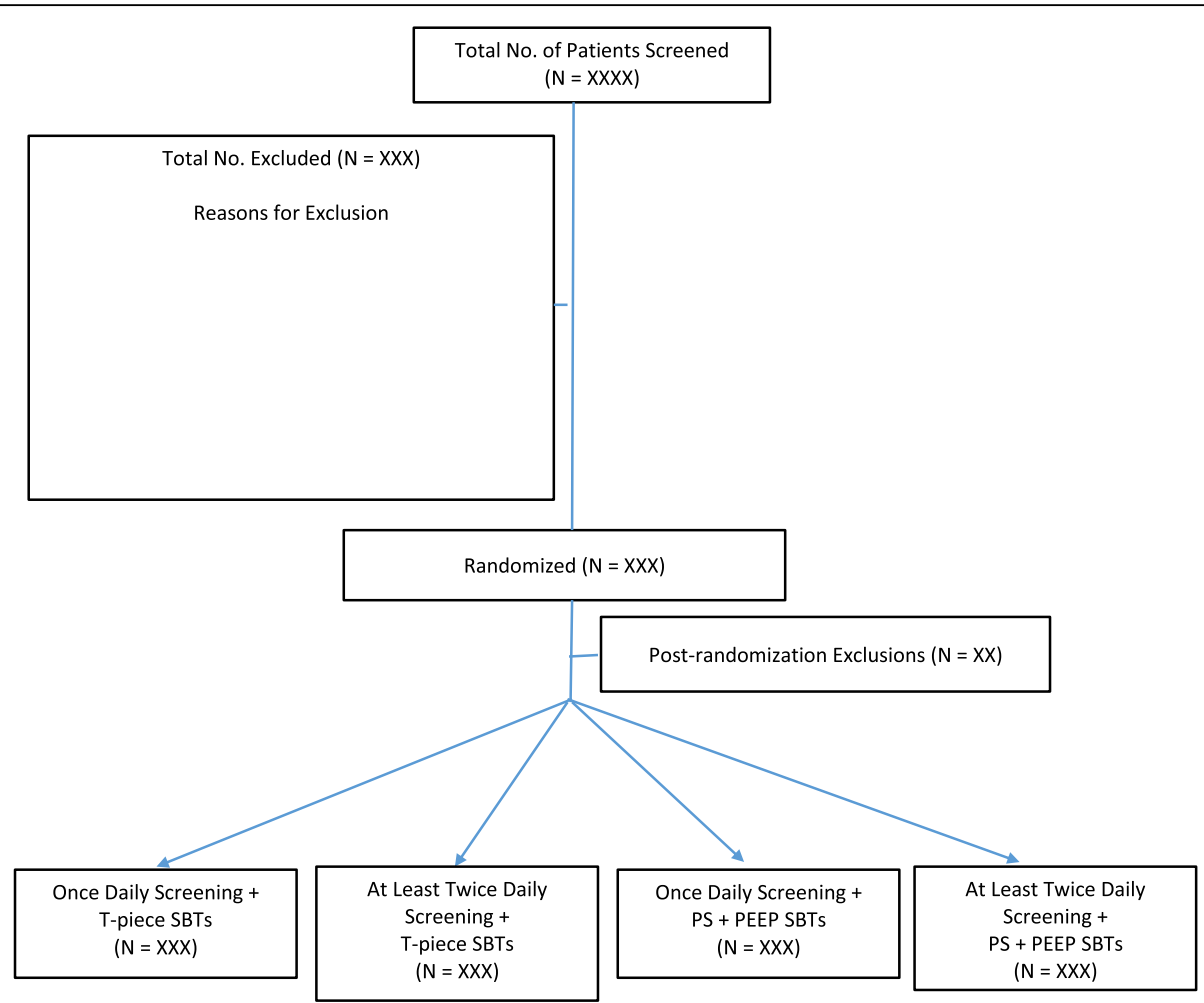

Fig. 1 Cumulative hazard and survival functions of patients infected by K. pneumoniae fitted to Lognormal distribution

PEEP) or with PS $>0$ and $\leq 8 \mathrm{~cm} \mathrm{H}_{2} \mathrm{O}$ with PEEP $>0$ and $\leq$ $5 \mathrm{~cm} \mathrm{H}_{2} \mathrm{O}$ [21]. Between SBT trials, patients will be returned to the mode of ventilation used before the SBT, unless criteria are met to remain on/return to a mode of support that assumes no spontaneous or triggered breaths (Additional file 1). We will use standardized criteria to determine SBT failure in both arms [27] (Table 2). After an unsuccessful SBT, patients will be returned to the ventilator settings used before the SBT and ventilator settings will be adjusted to restore respiratory comfort.

Table 2 Criteria for spontaneous breathing trial failure A failed SBT will be defined by the presence of any ONE of:

(1) A respiratory rate $>35$ breaths/min with signs of respiratory distress or an increase in respiratory rate $\geq 20 \%$ from baseline with signs of respiratory distress

(2) Oxygen saturation of arterial blood $\left(\mathrm{SaO}_{2}\right)$ or pulse oximetry $<90 \%$

(3) Heart rate $>140$ beats/min with signs of respiratory distress or an increase in $H R \geq 20 \%$ from baseline with signs of respiratory distress

(4) Systolic blood pressure $\geq 180$ or $\leq 90 \mathrm{mmHg}$

(5) The presence of somnolence, agitation, diaphoresis, or anxiety

(6) Requirement for the addition of or an increase in vasopressor or inotropic agent support

(7) Chest pain or other limiting pain precluding further continuation
Criteria to suspend the protocol and return to a controlled/ supported mode of ventilation

Patients should remain on a mode that permits spontaneous or triggered breaths between SBTs and at night. In all groups, patients will be permitted to return to/remain on a supported mode of ventilation without spontaneous or triggered breaths when one or more criteria are met (Table 3). Patients who meet any criteria will be reassessed daily to identify the earliest time when they meet initial inclusion criteria and the screening and SBT protocols can be resumed.

\section{Extubation}

Patients who pass an SBT will be assessed for extubation. Extubation should be performed as soon as possible after passing an SBT. To be extubated patients should meet all criteria depicted in Table 4 [27].

As this was not a trial focused on extubation, we did not protocolize extubation. Conversely, we will record the time that patients met criteria for extubation and the time patients were actually extubated.

\section{Other important considerations}

We standardized approaches to ventilator titration, use of NIV after extubation, reintubation, and tracheostomy [28, 29] (Additional file 1). 
Table 3 Criteria to suspend the protocol and return to a controlled/supported mode of ventilation

(1) Surgery or invasive procedures requiring sedation

(2) Respiratory distress as defined by:

a) sustained hypoxemia (pulse oximetry oxygen saturation $\left[\mathrm{SpO}_{2}\right]<$ 90\%) with an $\mathrm{FiO}_{2}>60 \%$ and PEEP $>10 \mathrm{~cm} \mathrm{H}_{2} \mathrm{O}$ or hypercapnia with $\mathrm{pH}<7.30$ OR clinical respiratory distress

b) repeated episodes ( $\geq 3$ episodes within $1 \mathrm{~h}$ ) wherein an inspiratory pressure (drive pressure + $\mathrm{PEEP}$ on pressure modes or plateau pressure on volume modes) of $35 \mathrm{~cm} \mathrm{H}_{2} \mathrm{O}$ or more is attained (despite suctioning, bronchodilation, etc.)

(3) Hemodynamic instability despite fluid boluses and requirement for high dose vasopressors: norepinephrine $>15 \mu \mathrm{g} / \mathrm{min}(0.2 \mu \mathrm{g} / \mathrm{kg} / \mathrm{min})$ or equivalent

(4) Suspected myocardial ischemia based on EKG and/or elevated Troponin I

(5) Neurologic deterioration with need to control $\mathrm{PaCO}_{2}$ (e.g. raised intracranial pressure) or central hypoventilation

(6) Respiratory rate $<10$ breaths/min related to need for increased sedation

(7) PEEP $\geq 13 \mathrm{~cm} \mathrm{H}_{2} \mathrm{O}$

(8) $\mathrm{FiO}_{2} \geq 71 \%$

$\mathrm{FiO}_{2}$ inspired fractional concentration of oxygen, $P E E P$ positive end-expiratory pressure, EKG electrocardiogram

\section{Follow-up}

Successful extubation is defined as the time when unsupported, spontaneous breathing began and was sustained for $\geq 48 \mathrm{~h}$ after extubation/disconnection in patients with tracheostomy [27]. We will collect daily data up to successful extubation, ICU death, ICU discharge, or until day 60 after randomization (deemed ventilator-dependent), whichever comes first. All patients will be followed to hospital discharge.

\section{Study outcomes \\ Primary outcome}

The primary outcome will be the time to successful extubation.

Table 4 Extubation criteria

(1) $\mathrm{SpO}_{2} \geq 90 \%$ or at baseline level in chronically hypoxemic patients on an $\mathrm{FiO}_{2} \leq 40 \%$ and PEEP $\leq 5 \mathrm{~cm} \mathrm{H} \mathrm{O}$

(2) A cough of sufficient strength to clear secretions and must not require suctioning more than every $2 \mathrm{~h}$

(3) Patients should be hemodynamically stable (off vasopressors or on minimal levophed, i.e. $\leq 7 \mu \mathrm{g} / \mathrm{min}[0.1 \mu \mathrm{g} / \mathrm{kg} / \mathrm{min}$ or equivalent])

(4) A level of consciousness sufficient to ensure airway protection and

(5) A cuff leak is present

All of the above criteria (except nos. 4 and 5) will also apply to patients who undergo trach mask trials and are disconnected.

$\mathrm{SpO}_{2}$ pulse oximetry saturation, $\mathrm{FiO}_{2}$ fraction of inspired oxygen concentration, PEEP positive end-expiratory pressure

\section{Secondary outcomes}

Secondary outcomes will include: (1) ICU mortality; (2) hospital and 90-day mortality [30]; (3) time to first passing an SBT; (4) total duration of mechanical ventilation (invasive and non-invasive); (5) ICU length of stay; (6) hospital length of stay; (7) use of NIV after extubation [15, 31]; (8) VAP; (9) adverse events (AEs), self-extubation, tracheostomy [28, 29], reintubation, prolonged ventilation (patients who remain intubated at day 14 and/or day 21), ICU readmission [32, 33]; (10) proportion of patients who receive sedation, analgesia, antipsychotics at key time points; (11) proportion who screen positive for delirium at key time points [34-37], (12) HRQoL (EuroQuol EQ-5D) six months after randomization [38, 39]; and (13) functional status six months after randomization using the Functional Independence Measure (FIM) [40, 41].

\section{Analytic plan}

We will summarize baseline data using descriptive statistics.[42]. All analyses will be performed adhering to the intention-to-treat principle.

\section{Primary analysis}

Time-to event outcomes present special challenges because death is a competing risk and survivor-only analyses are improper sub-groups. We will construct cumulative incidence curves to provide outcome estimates accounting for death for screening frequency and SBT technique. Cause-specific treatment effects will be depicted with hazard ratios (HR) with 95\% confidence intervals (CI) from Cox models.

\section{Secondary analysis}

We will report treatment effects in time to event analyses using $\mathrm{HR}$ and odds ratio (OR) with 95\% CIs for binary outcomes and mean difference with 95\% CI for continuous outcomes [42].

\section{Exploratory and adjusted analyses}

To assess the effect of age (continuous variable) by treatment interaction on the HR of time to successful extubation, we will construct a Cox regression model using a restricted cubic spline for age. Instead of arbitrarily assigning different levels for each age group, period, and cohort, we will create a smoothing function or spline (collections of cubic polynomials joined smoothly at a predefined number of points [knots]). The number of knots is expected to be between three and five but will be selected based on the sample size assuming that the relationship with age will change gradually and smoothly. We will evaluate fit using bootstrap techniques. This technique allows for non-linearities and interactions between variables that are more flexible than 
the linear contrasts traditionally used in regression models and is easier to depict and interpret [43]. In exploratory and adjusted analyses, we will assess for an interaction between screening frequency and SBT technique and variables (e.g. COPD, frailty etc.) of potential prognostic importance.

\section{Interim analyses}

Interim analyses for safety (AEs) and efficacy (primary outcome) will be performed at $25 \%, 50 \%$, and $75 \%$ of accrual and reviewed by the Data Safety and Monitoring Committee (DSMB). Given the potential risk of stopping early for benefit, statistical significance will be declared using small $p$ values according to the O'Brien-Fleming boundaries for the primary outcome and reintubation rate [44].

\section{Sample size}

To compute sample size and take into consideration deaths that occur before successful extubation, we used cumulative incidence curves generated from our pilot trials and computed three mortality HRs per patient day (HR 2.9 [Release Trial] and HR 3.3 [SENIOR Trial] and HR 3.2 [combined]) [25]. We will require 760 patients to demonstrate a reduction in time to successful extubation from a median of 5.0 days to 4.0 days (HR 1.25) [27, 45] with $80 \%$ power and $\alpha=0.05$ and allowing for three interim analyses. A priori, we do not expect an interaction since mechanistically and sequentially an interaction is unlikely. Since the groups are orthogonal, the main effects (in the absence of interactions) will have the same power to detect the same size differences.

\section{Trial organization}

The Applied Health Research Centre (AHRC; www.ahr conline.com) of St. Michaels Hospital will serve as the data management and coordinating center. The AHRC is a not-for profit academic research organization affiliated with the University of Toronto. The Methods Centre will be responsible for data management and analysis as well as providing progress and data reports to the Steering Committee and DSMB.

The FAST-NAWC is being implemented with the input of ICU survivors and family members of former ICU patients from Canada and the USA. Our Patient and Family Advisory Committee (PFAC) members have direct experience with mechanical ventilation. The PFAC will serve in an advisory capacity to the Steering Committee. During protocol development, our PFAC members aided in identifying our primary outcome and advocated to include a six-month follow-up study. All PFAC members provided letters of support for grant submissions and one PFAC member reviewed a portion of the grant [46]. During trial implementation, PFAC members will: (1) be represented on the trial Steering Committee and DSMB; (2) directly advised the Steering Committee on study design and implementation issues; (3) assist with preparation of study materials; (4) aid in selecting a metric to assess functional status at sixmonth follow-up assessments; (5) participate in bimonthly teleconferences; and (6) contribute to developing a moderated on-line space that will serve as a repository for patient and family narratives [46].

\section{Trial status}

The FAST PILOT trial was launched on 15 June 2016 in 11 ICUs and was completed on 8 December 2018 [47]. After received full funding to conduct the FAST NAWC (protocol version 5; 4 May 2018), we re-launched the factorial design RCT on 18 January 2018. We anticipate recruitment will be completed by March 2021.

\section{Discussion}

The FAST-NAWC Trial is novel in several ways. First, the FAST-NAWC Trial will be the largest weaning trial conducted in the North America where weaning involves close collaboration between RTs and physicians. Second, the FAST-NAWC seeks to identify the optimal SBT screening frequency and SBT technique to minimize patients' exposure to invasive ventilation and ventilator-related complications. Third, this trial is being implemented with novel collaborations between Canadian and American research networks and respiratory care and critical care societies. Finally, the FAST-NAWC Trial is being conducted with the input of ICU survivors and family members. They will ensure that the trial is implemented in a manner that is sensitive to patient's and families' needs.

To address the concerns that elderly, critically ill patients may experience more AEs with more frequent SBT screening and be less likely to be enrolled in this trial due to concomitant treatment limitations and comorbidities, we conducted two parallel, multicenter, pilot screening frequency trials [25]. Both trials compared OD with ALTD screening in elderly patients aged $\geq 65$ years (SENIOR Trial; ClinicalTrials.gov NCT022434 49; 11 ICUs) and non-elderly patients aged $<65$ years (RELEASE Trial; NCT02001220; 10 ICUs) [25]. Recognizing the need to change screening culture, we prioritized the evaluation of SBT screening frequency and permitted centers to use their preferred SBT technique in both pilot trials. We demonstrated similar recruitment and consent rates, few AEs, and comparable outcomes in younger and older patients [25]. Conduct of the pilot trials enabled us to refine several exclusion criteria and enhance the generalizability of our findings. Subsequently, we conducted a factorial design, FAST pilot trial $(n=110)$ comparing both screening frequency 
and SBT technique [48]. With this trial, we refined recruitment estimates, identified barriers to recruitment, and assessed potential co-interventions. Patients enrolled in the FAST pilot trial will be included in the FASTNAWC trial.

Careful consideration has been given to important aspects of the FAST-NAWC study design to limit selection, identification, treatment, and performance bias in this necessarily unblinded weaning trial. To limit selection bias, we will use a central randomization process with full allocation concealment. To limit identification bias, RTs will conduct SBT screening assessments and SBTs. To limit delays in identifying SBT candidates, we avoided use of subjective assessments in selecting our SBT screening criteria (e.g. level of consciousness, no sedation, no vasopressors, hemoglobin > $100 \mathrm{~g} / \mathrm{L}$ ). Conversely, we included an objective test, the rapid shallow breathing index (f/VT) [26], measured on standardized settings, in all SBT screening assessments. We will record practices before SBT screening that have the potential to influence SBT performance including pain, sedation, and delirium management and whether patients are being mobilized. To enhance the generalizability of our findings, we will permit SBTs to be $30-120 \mathrm{~min}$ in duration at clinician's discretion. Finally, to limit treatment bias, we provided guidance on: (1) titration of ventilator settings, PEEP, and $\mathrm{FiO}_{2}$; (2) use of NIV after extubation; (3) reintubation; and (4) tracheostomy.

Establishing the optimal screening frequency and SBT technique is appealing to ICU clinicians because these interventions are sensible, low-risk, and represent an efficient use of current resources. Findings from this trial have the potential to change clinical practice, enhance the care delivered to critically ill adults, and improve outcomes.

\section{Additional file}

Additional file 1: Additional Protocol Information. (DOCX 25 kb)

\section{Acknowledgments}

We wish to thank the members of the Canadian Critical Care Trials Group for their methodologic advice and content expertise and Dr. Lauralyn Mclntyre for her review of the protocol.

\section{Guarantor}

Dr. Burns will be the guarantor.

\section{Study sponsor}

The funder had no involvement in study design; collection, management, analysis, and interpretation of data; writing of the protocol; and the decision to submit the protocol for publication.

\section{Authors' contributions}

Study design: all authors. Study funding: KB, LR, DC, AS, BR, FL, JD, PD, MM, AG, PK, SM, KK, NH. Manuscript preparation: all authors. Manuscript approval: all authors (including members of the Patient and Family Advisory Committee). All authors read and approved the final manuscript.

\section{Funding}

The FAST-NAWC is funded by the Canadian Institutes of Health Research.

\section{Availability of data and materials}

There are no data related to the protocol manuscript.

\section{Ethics approval}

This protocol was approved by the research ethics board of St. Michael's Hospital (Toronto) and of participating ICUs. Informed consent will be obtained from trial participants as per the requirements of participating research ethics boards.

\section{Consent for publication}

Not applicable.

\section{Competing interests}

The authors declare that they have no competing interests.

\section{Author details}

${ }^{1}$ Interdepartmental Division of Critical Care, University of Toronto, Toronto, ON, Canada. ${ }^{2}$ Department of Medicine, Division of Critical Care Medicine, St Michael's Hospital, 30 Bond Street, Office 4-045 Donnelly Wing, Toronto, ON M5B 1W8, Canada. 'i Ka Shing Knowledge Institute, St. Michael's Hospital, 30 Bond Street, Office 4-045 Donnelly Wing, Toronto, ON M5B 1W8, Canada. ${ }^{4}$ Department of Health Research Methods, Evidence and Impact, McMaster University, Hamilton, ON, Canada. ${ }^{5}$ Ottawa Hospital Research Institute, University of Ottawa, Ottawa, ON, Canada. ${ }^{6}$ Department of Medicine, McMaster University, Hamilton, ON, Canada. ${ }^{7}$ Centre de Recherche du Centre Hospitalier Universitaire de Sherbrooke, Sherbrooke, QC, Canada. ${ }^{8}$ School of Pharmacy, Northeastern University, Boston, MA, USA. 'Division of Pulmonary, Critical Care and Sleep Medicine, Tufts Medical Center, Boston, MA, USA.

${ }^{10}$ Centre for Health Evaluation and Outcome Sciences, Vancouver, BC,

Canada. ${ }^{11}$ University of British Columbia, Vancouver, BC, Canada. ${ }^{12} \mathrm{Critical}$ Care Medicine, Longbeach Memorial, Longbeach, CA, USA. ${ }^{13}$ Patient and Family Advisory Committee Member, FAST - NAWC Trial, Toronto, Canada.

Received: 16 May 2019 Accepted: 8 August 2019

Published online: 11 October 2019

\section{References}

1. Esteban A, Alia I, Ibanez J, Benito S, Tobin MJ. Modes of mechanical ventilation and weaning. A national survey of Spanish hospitals. The Spanish Lung Failure Collaborative Group. Chest. 1994;106:1188-93.

2. Esteban A, Anzueto A, Frutos F, et al. Characteristics and outcomes in adult patients receiving mechanical ventilation: a 28-day international study. JAMA. 2002;287(3):345-55.

3. Pingleton SK. Complications of acute respiratory failure. Am Rev Respir Dis. 1988;137:1463-93.

4. Heyland DK, Cook DJ, Griffith L, Keenan SP, Brun-Buisson C. The attributable morbidity and mortality of ventilator associated pneumonia in the critically ill patient. The Canadian Critical Care Trials Group. Am J Respir Crit Care Med. 1999:159:1249-56.

5. Thille AW, Harrois A, Schortgen F, Brun-Buisson C, Brochard L. Outcomes of extubation failure in medical intensive care unit patients. Crit Care Med. 2011;39:2612-8.

6. Esteban A, Alia I, Gordo F, et al. Extubation outcome after spontaneous breathing trials with t-tube or pressure support ventilation. Am J Respir Crit Care Med. 1997;156:459-65.

7. Esteban A, Alia I, Tobin MJ. Effect of spontaneous breathing trial duration on outcome of attempts to discontinue mechanical ventilation. Am J Respir Crit Care Med. 1999;159:512-8.

8. Maclntyre NR, Cook DJ, Ely EW Jr, Epstein SK, Fink JB, Heffner JE, et al. Evidence based guidelines for weaning and discontinuing ventilatory support. A collective task force facilitated by the American College of Chest Physicians, the American Association for Respiratory Care and the College of Critical Care Medicine. Chest. 2001;120(Supple 6):375S-95S.

9. Blackwood B, Burns K, Cardwell C, Lavery G, O'Halloran P. Use of weaning protocols for reducing duration of mechanical ventilation in critically ill 
adult patients: Un updated Cochrane systematic review and meta-analysis. Cochrane Database Syst Rev. 2014;Issue 11:CD006904. https://doi.org/10.1 002/14651858.CD006904.

10. Perren A, Domenighetti G, Mauri S, et al. Protocol-directed weaning from mechanical ventilation; clinical outcome in patients randomized for a 30-minute and 120-minute trial with pressure support. Intensive Care Med. 2002;28:1058-63.

11. Burns KE, Soliman I, Adhikari NKJ, Zwein A, Wong JTY, Gomez-Builes C, et al. Trials directly comparing alternative spontaneous breathing trial techniques: a systematic review and meta-analysis. Crit Care. 2017;21(1):127.

12. Girard TD, Alhazzani W, Kress JP, Ouellette DR, Schmidt GA, Truwit JD, et al. An Official American Thoracic Society/American College of Chest Physicians Clinical Practice Guideline: Liberation from Mechanical Ventilation in Critically III Adults Rehabilitation Protocols, Ventilator Liberation Protocols, and Cuff Leak Tests. Am J Respir Crit Care Med. 2017;195(1):120-33.

13. Brochard L, Rauss A, Benito S, et al. Comparison of three methods of gradual withdrawal from ventilatory support during weaning from mechanical ventilation. Am J Respir Crit Care Med. 1994;150:896-903.

14. Esteban A, Frutos F, Tobin MJ, et al. A comparison of four methods of weaning patients from mechanical ventilation. N Engl J Med. 1995; 332:345-50

15. Marelich GP, Murin S, Battistella F, et al. Protocol weaning of mechanical ventilation in medical and surgical patients by respiratory care practitioners and nurses. Effect on weaning time and incidence of ventilator associated pneumonia. Chest. 2000;118:459-67.

16. Burns KEA, Lellouche F, Loisel F, Slutsky AS, Meret A, Smith O, et al. Weaning critically ill adults from invasive mechanical ventilation: a national survey. Can J Anaesth. 2009;56(8):567-76.

17. Burns KE, Raptis SR, Nisenbaum R, Rizvi L, Jones A, Bashik J, et al. International practice variation in weaning critically ill adults from invasive mechanical ventilation. Ann Am Thorac Soc. 2018;15(4):494-502. https://doi. org/10.1513/AnnalsATS.201705-4100C.

18. Godard S, Herry C, Westergaard P, Scales N, Brown SM, Burns K, et al. Practice variation in spontaneous breathing trial performance and reporting. Can Respir J. 2016;2016:9848942. https://doi.org/10.1155/2016/9848942.

19. Ladiera MT, Vital FM, Andriolo RB, Andriolo BN, Attalah AN, Peccin MS. Pressure Support vs. T-tube for weaning from mechanical ventilation. Cochrane Database Syst Rev. 2014;Issue 5:CD006056.

20. Pellegrini JA, Moraes RB, Maccari JG, de Oliveira RP, Savi A, Ribeiro RA, et al. Spontaneous breathing trials with T-piece or pressure support ventilation. Respir Care. 2016;61(12):1693-703.

21. Boles JM, Bion J, Connors A, Herridge M, Marsh B, Melot C, Pearl R, et al. Task Force: weaning from mechanical ventilation. Eur Respir J. 2007;29:1033-56.

22. Sklar MC, Burns K, Rittayamai N, Lanys A, Rauseo M, Chen L, et al. Effort to breathe with various spontaneous breathing trial techniques. A physiological meta-analysis. Am J Respir Crit Care Med. 2017;195(11):147785. https://doi.org/10.1164/rccm.201607-13380C.

23. Burns KE, Jacob SK, Aguirre V, Gomes J, Mehta S, Rizvi L. Stakeholder engagement in trial design: survey of visitors to critically ill patients regarding preferences for outcomes and treatment options during weaning from mechanical ventilation. Ann Am Thorac Soc. 2016;13(11):1962-8.

24. Tri-Council Policy Working Party on Ethics. Code of ethical conduct for research involving humans. Ottawa. The Medical Research Council of Canada, the Natural Sciences and Engineering Research Council of Canada and the Social Sciences and Humanities Research Council of Canada, 1997 www.ncehr-cnehr.org/english/code_2. Accessed 11 Feb 2016).

25. Burns KEA, Wong JTY, Dodek P, Cook DJ, Lamontagne F, Cohen A, et al. Frequency of screening for weaning from mechanical ventilation: two contemporaneous proof-of-principle randomized controlled trials. (In Press: Critical Care Medicine). Crit Care Med. 2019:47:817-25. https://doi.org/10.1 097/CCM.0000000000003722

26. Yang $\mathrm{KL}$, Tobin MJ. A prospective study of indexes predicting the outcome of trials of weaning from mechanical ventilation. N Engl J Med. 1991;324:1445-50.

27. Burns KE, Meade MO, Lessard MR, Hand L, Zhou Q, Keenan SP, et al. Wean Earlier and Automatically with New Technology (The WEAN Study): a multicentre pilot RCT. Am J Respir Crit Care Med. 2013;187(11):1203-11.

28. Terragni PP, Antonelli M, Fumagalli R, Faggiano C, Berardino M, Pallavicini $\mathrm{FB}$, et al. Early vs. late tracheotomy for prevention of pneumonia in mechanically ventilated adult ICU patients: a randomized controlled trial. JAMA. 2010;303(15):1483-9.

29. Young D, Harrison DA, Cuthbertson BH, Rowan K, for the Trachman Collaborators. Effect of early vs. late tracheostomy placement on survival in patients receiving mechanical ventilation: the TracMan randomized trial. JAMA. 2013;309(20):2121-9.

30. Nisula A, Kaukonen KM, Vaara ST, Korhonen AM, Poukkanen M, Karlsson S, et al. Incidence, risk factors and 90-day mortality of patients with acute kidney injury in Finnish intensive care units: the FINNAKI study. Intensive Care Med. 2013;39(3):420-8.

31. Burns KEA, Premji A, Meade MO, Adhikari NKJ. Noninvasive ventilation as a weaning strategy for mechanical ventilation in adults with respiratory failure: a Cochrane systematic review. CMAJ. 2014;186(3):E112-22.

32. Frost SA, Alexandrou E, Bogdanovski T, Salamonson Y, Davidson PM, Parr MJ, et al. Severity of illness and risk of readmission to intensive care: a meta -analysis. Resuscitation. 2009;80(5):505-10. https://doi.org/10.1016/j. Resuscitation.2009.02.015.

33. Rosenberg AL, Watts C. Patients readmitted to ICUs: a systematic review of risk factors and outcomes. Chest. 2000;118(2):492-502.

34. Riker RR, Picard JT, Fraser GL. Prospective evaluation of the Sedation-Agitation Scale for adult critically ill patients. Crit Care Med. 1999;27(7):1325-9.

35. Sessler CN, Gosnell MS, Grap MJ, Brophy GM, O'Neal PV, Keane KA, et al. The Richmond Agitation-Sedation Scale: validity and reliability in adult intensive care unit patients. Am J Respir Crit Care Med. 2002;166(10):1338-44.

36. Devlin JW, Brummel NE, Al-Qadheeb NS. Optimizing the recognition of delirium in the intensive care unit. Best Pract Res Clin Anaesthesiol. 2012;26:385-93.

37. Morris PE, Goad A, Thompson C, Taylor K, Harry B, Passmore L, et al. Early intensive care unit mobility therapy in the treatment of acute respiratory failure. Crit Care Med. 2008;36:2238-43.

38. Herdman M, Gudex C, Lloyd A, et al. Development and preliminary testing of the new five-level version of EQ-5D (EQ-5D-5L). Qual Life Res. 2011;20:1727-36.

39. Weiss DS. The Impact of Event Scale: Revised. In: Wilson JP, Tang CS, editors. Cross-cultural assessment of psychological trauma and PTSD. New York: Springer; 2007. p. 219-38. Polisher Research Institute. Instrumental Activities of Daily Living Scale (IADL). http://www.abramsoncenter.org/PRI/ documents/IADL.pdf. Accessed 19 Oct 2015.

40. Oczkowski WJ, Barreca S. The functional independence measure: its use to identify rehabilitation needs in stroke survivors. Arch Phys Med Rehabil. 1993;74:1291-4

41. Koyama T, Matsumoto K, Okuno T, Domen K. Relationships between independence level of single motor-FIM items and FIM-motor scores in patients with hemiplegia after stroke: an ordinal logistic modelling study. J Rehabil Med. 2006;38:280-6.

42. Streiner DL, Norman GR. Health measurement scales - a practical guide to their development and use. 4th ed. Oxford: Oxford University Press; 2008.

43. Fan J, Yao Q. "Spline Methods". Nonlinear time series: nonparametric and parametric methods. New York: Springer; 2005. p. 247.

44. O'Brien PC, Fleming TR. A multiple testing procedure for clinical trials. Biometrics. 1979;35:549-56

45. Lellouche F, Mancebo J, Jolliet P, Roeseler J, Schortgen F, Dojat M, et al. A multicenter randomized trial of computer-driven protocolized weaning from mechanical ventilation. Am J Respir Crit Care Med. 2006;174:894-900.

46. Burns KE, Devlin J, Hill N. Patient and family engagement in designing and implementing a weaning trial: a novel research paradigm in critical care. Chest. 2017;152(4):707-11.

47. Burns KEA, Rizvi L, Dodek P, Lamontagne F, Seely AJE, et al. (2016) Frequency of Screening and SBT Technique Trial. J Clin Trials 6:284. https:// doi.org/10.4172/2167-0870.1000284

48. Burns KEA, Rizvi L, Dodek P, Lamontagne F, Seely AJE, Rochwerg B, et al. Frequency of screening and 'Spontaneous Breathing Trial' Technique Study (The FAST Trial): design of a multicentre, pilot, factorial randomized controlled trial. Clin Trials. 2016;6:284.

\section{Publisher's Note}

Springer Nature remains neutral with regard to jurisdictional claims in published maps and institutional affiliations. 